\title{
Heart size in thyrotoxicosis
}

\author{
J. S. StafFurth \\ M.D., F.R.C.P. \\ N. D. W. MORRISON \\ D.M.R.D. \\ Consultant Physician \\ Consultant Radiologist
}

Lewisham Hospital, London, S.E.13

\section{Summary}

(1) Enlargement of the heart was found in $35 \%$ of a group of older patients with thyrotoxicosis who had no evidence of associated heart disease. Atrial fibrillation was present in $48 \%$ of patients with cardiac enlargement and in $10 \%$ with a normal sized heart. Both complications were more common when there was associated heart disease.

(2) In patients with an enlarged heart but no associated heart disease a reduction in heart-size after treatment of thyrotoxicosis was observed in five of twenty-three who had sinus rhythm throughout, and in three of eleven who had atrial fibrillation on presentation with subsequent reversion to sinus rhythm. There was no reduction in heart size in ten who had persistent atrial fibrillation after completion of treatment. Reduction in heart size was most marked in patients who had presented with frank cardiac failure.

(3) The incidence of cardiac enlargement and atrial fibrillation both increased with advancing age but whereas enlargement was common over 45 years atrial fibrillation was rarely seen under 55 years of age. Only one of thirteen patients with an enlarged heart under 55 years had atrial fibrillation.

(4) It is concluded that enlargement of the heart may be a manifestation of thyrotoxic heart disease, that it may precede the onset of atrial fibrillation in some instances, and consequently that atrial fibrillation is not necessarily the cause of an enlarged heart in thyrotoxicosis.

\section{Introduction}

Although the heart is involved from the onset in most patients with thyrotoxicosis since sinus tachycardia is one of the cardinal signs, the term Thyrotoxic Heart Disease is usually reserved for patients with atrial fibrillation (AF) or congestive cardiac failure (Wood, 1961). Enlargement of the heart with sinus rhythm may also occur in thyrotoxicosis but this is not so widely recognized, though it is quite common (Parkinson \& Cookson, 1931). There is surpris- ingly little information on any change in the size of the heart that may occur after treatment of the thyrotoxicosis whether sinus rhythm or AF was present originally.

In this study the chest $X$-rays have been examined retrospectively in a selected series of patients with thyrotoxicosis who were referred for treatment with radioactive iodine, and where possible, these have been compared with $\mathrm{X}$-rays after completion of treatment when the patients were euthyroid.

\section{Subjects and methods}

The patients have been drawn from 239 confirmed cases of thyrotoxicosis who were treated with radioactive iodine in Lewisham Hospital from 1958 to 1965 . In some instances, the first chest X-ray and preliminary treatment had been performed at other hospitals in the area. The subjects are a selected group because only older patients are normally accepted for this treatment, and many of them were particularly referred because of the presence of heart disease. Many of the subjects have been included in other reports from our department dealing with different aspects of management with radioactive iodine (Staffurth, Gibberd \& Hilton, 1965; Staffurth \& Young, 1967).

The heart size has been measured direct on standard PA chest X-rays at $6 \mathrm{ft}$. The transverse diameter of the heart (t.d.) was determined as the sum of the greatest distances from the mid-line to the right and left cardiac borders, care being taken to exclude epipericardial fat and any other non-cardiac shadow. The internal transverse diameter (i.d.) of the thorax was measured as the greatest distance between the inner borders of the ribs. The cardio-thoracic ratio (CTR) was determined by expressing t.d. as a percentage of i.d. (Lusted \& Keats, 1967). For descriptive purposes the CTR has arbitarily been considered to be normal when less than $50 \%$. In the follow-up X-rays, which were taken at varying intervals between 6 months and 5 years after treatment, a difference in t.d. of $1.0 \mathrm{~cm}$ or 
less has not been considered a significant change.

Hypertension has been considered to be present in any patient in whom the diastolic blood pressure before treatment was $100 \mathrm{mmHg}$ or more. Ischaemic heart disease has been diagnosed when either there was a history of angina pectoris or a frank myocardial infarct; or if there were unequivocal changes on the electrocardiogram of $S-T$ depression, $T$ wave inversion or bundle branch block (Sandler \& Wilson, 1959 ; Summers \& Surtees, 1961).

\section{Results}

Satisfactory chest X-rays were available for assessment in 175 patients, of whom twenty-two had hypertension, sixteen had ischaemic heart disease, eight had rheumatic heart disease, one had a ventricular septal defect and one had severe anaemia. In the remainder an original film had never been taken, it had been destroyed or mislaid, or it had to be rejected because of deformity of the chest wall.

Heart size before and after treatment of thyrotoxicosis in cases without associated heart disease (see Table 1)

There were 127 patients in whom thyrotoxicosis was the only clinical cause of heart disease and in eighty-three the heart was normal in size. Follow-up chest $\mathrm{X}$-rays were not requested in the seventy-five subjects with sinus rhythm and a normal sized heart. In eight subjects with AF and a normal sized heart there was no change in size after treatment in the six instances where a second film was available, whether or not they had reverted to sinus rhythm.

The heart was enlarged in forty-four cases, of whom twenty-one had AF and twenty-three were in sinus rhythm. Following treatment eleven of the patients with AF reverted to sinus rhythm, in five of whom there was no change in heart size ; in three there was a marked reduction in size and in three there was further increase in size, all of whom had gained a considerable amount of weight. None of the patients with persistent AF after treatment showed any reduction in heart size. Following treatment of those in sinus rhythm, there was no change in heart size in thirteen, it became moderately smaller in three and markedly smaller in two. One of the two patients who had a marked diminution in heart size following treatment had presented with frank cardiac failure (R.L., Table 3 ) but in the other, a female aged 50 who had severe thyrotoxicosis, there had been only moderate exertional dyspnoea. In another patient with sinus rhythm throughout, who had had a prior chest X-ray for an unrelated condition, there was an increase in t.d. of $1.5 \mathrm{~cm}$ in 6 months with the development of thyrotoxicosis and a return to the original heart size after treatment.

Heart size before and after treatment of thyrotoxicosis in cases with associated hypertension or ischaemic heart disease

There were thirty-eight patients with thyrotoxicosis who had hypertension, ischaemic heart disease or both, of whom twelve, two with AF, had a normal sized heart. The heart was en larged in twenty-six, of whom fourteen had AF? and twelve were in sinus rhythm. Any changes in heart size in these patients is summarized in Table 2. A comparison of the two tables shows that relatively more subjects with associated heart

TABLE 1

Change in heart size after treatment of thyrotoxicosis in patients without associated heart disease

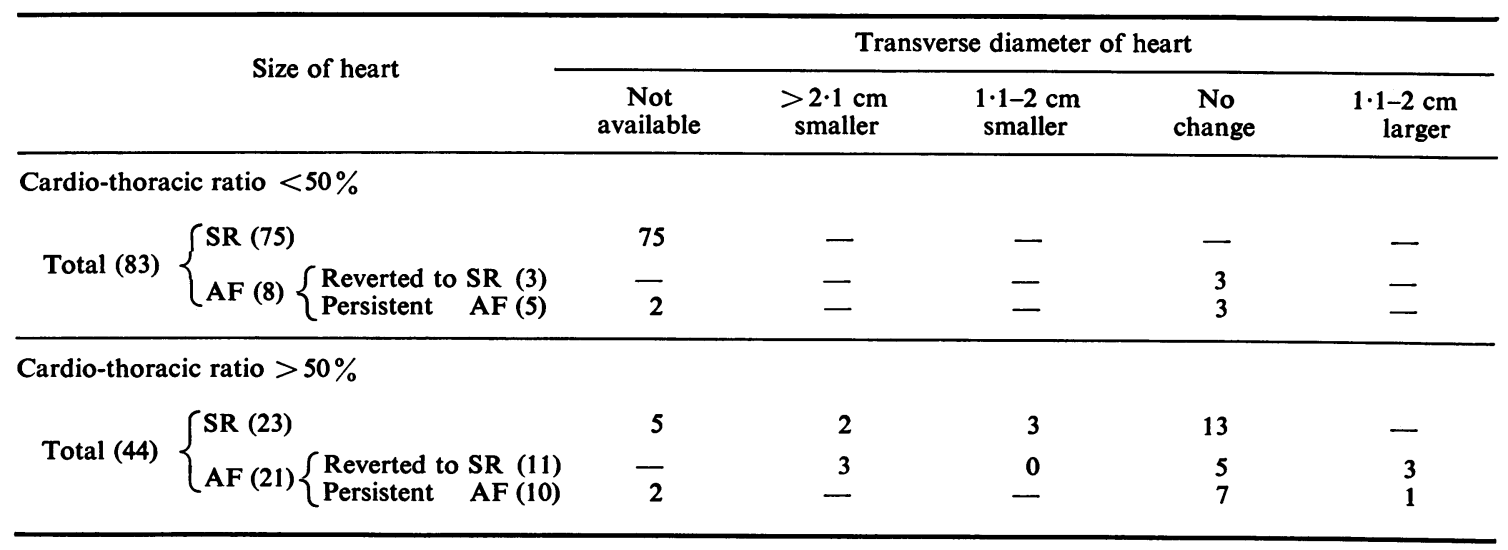

SR = Sinus rhythm; AF = Atrial fibrillation. 
TABLE 2

Change in heart size after treatment of thyrotoxicosis in patients with associated hypertension or ischaemic heart disease

\begin{tabular}{|c|c|c|c|c|c|c|c|}
\hline \multirow{2}{*}{\multicolumn{3}{|c|}{ Size of heart }} & \multicolumn{5}{|c|}{ Transverse diameter of heart } \\
\hline & & & $\begin{array}{c}\text { Not } \\
\text { available }\end{array}$ & $\begin{array}{l}>2 \cdot 1 \mathrm{~cm} \\
\text { smaller }\end{array}$ & $\begin{array}{l}1 \cdot 1-2 \mathrm{~cm} \\
\text { smaller }\end{array}$ & $\begin{array}{c}\text { No } \\
\text { change }\end{array}$ & $\begin{array}{c}1 \cdot 1-2 \mathrm{~cm} \\
\text { larger }\end{array}$ \\
\hline \multicolumn{8}{|c|}{ Cardio-thoracic ratio $<50 \%$} \\
\hline \multirow{2}{*}{ Total (12) } & $\int \mathrm{SR}(10)$ & & 7 & - & - & 3 & - \\
\hline & AF (2) & $\left\{\begin{array}{l}\text { Reverted to SR (2) } \\
\text { Persistent AF (0) }\end{array}\right.$ & 2 & - & - & - & - \\
\hline \multicolumn{8}{|c|}{ Cardio-thoracic ratio $>50 \%$} \\
\hline \multirow{2}{*}{ Total (26) } & $\int S R(12)$ & & 1 & - & 1 & 9 & 1 \\
\hline & AF (14) & $\left\{\begin{array}{l}\text { Reverted to SR (8) } \\
\text { Persistent AF (6) }\end{array}\right.$ & $\begin{array}{l}2 \\
3\end{array}$ & - & 1 & $\begin{array}{l}5 \\
2\end{array}$ & $-\overline{1}$ \\
\hline
\end{tabular}

SR = Sinus rhythm; AF = Atrial fibrillation.

disease had cardiac enlargement and AF, but that the proportion with AF who reverted to sinus rhythm when euthyroid was approximately the same in both groups, irrespective of the original size of the heart.

Changes in heart size in severe cardiac failure (Table 3)

Ten patients had severe cardiac failure on admission to hospital. In four this was due to thyrotoxicosis only, two had associated hypertension and four had mitral valve disease. In seven of them there was a marked reduction in heart size when they were euthyroid, in one there was no change, and in two the heart became larger. In five of six cases where a second chest $X$-ray had been taken after treatment of the cardiac failure, but before effective treatment of thyrotoxicosis, there was a marked reduction in heart size; and in four of these there was a further reduction in size when they were euthyroid in spite of persistent $\mathrm{AF}$ in three.

Relationship of age and blood pressure to heart size and atrial fibrillation

The age distribution of the patients without associated heart disease is shown in Fig. 1. Under 45 years there was one subject with an enlarged heart but there was no other instance in twenty additional random cases under 45 years who had been treated by thyroidectomy. Between 45 and 55 years twelve of thirty-seven patients had enlargement of the heart, only one of whom had AF. Thereafter there was a progressive increase in the proportion of patients having an enlarged heart with advancing age and a similar pro- portional increase in the incidence of $\mathrm{AF}$, which was uncommon under 55 years.

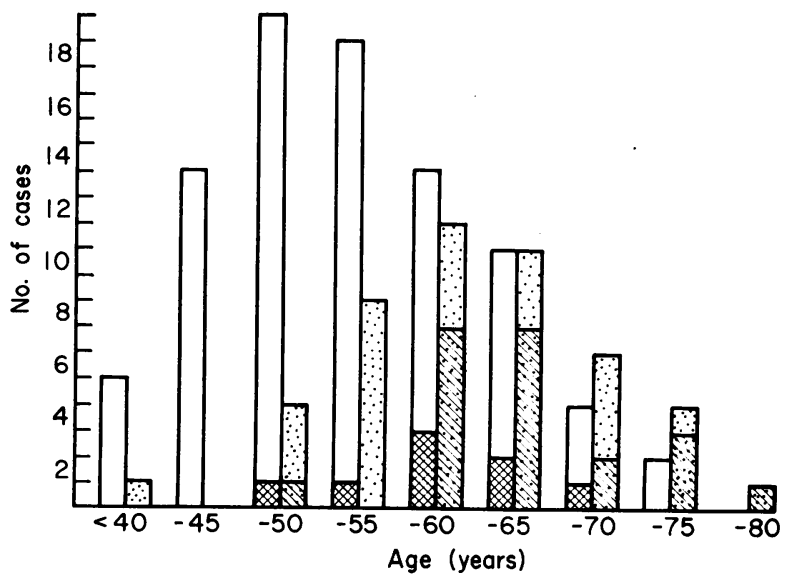

FIG. 1. Age distribution of 127 thyrotoxic patients without associated heart disease according to heart size and presence of atrial fibrillation. Open columns, Normal heart; stippled columns, enlarged heart; cross-hatched columns, atrial fibrillation.

The distribution of the systolic blood pressures in these same patients when they presented with thyrotoxicosis is shown in Fig. 2 from which no definite trend can be discerned. On the whole there is a higher incidence of cardiomegaly in patients with a blood pressure greater than $160 \mathrm{mmHg}$, but there are many instances of enlargement when the systolic blood pressure was less than this. Further the distribution of the 


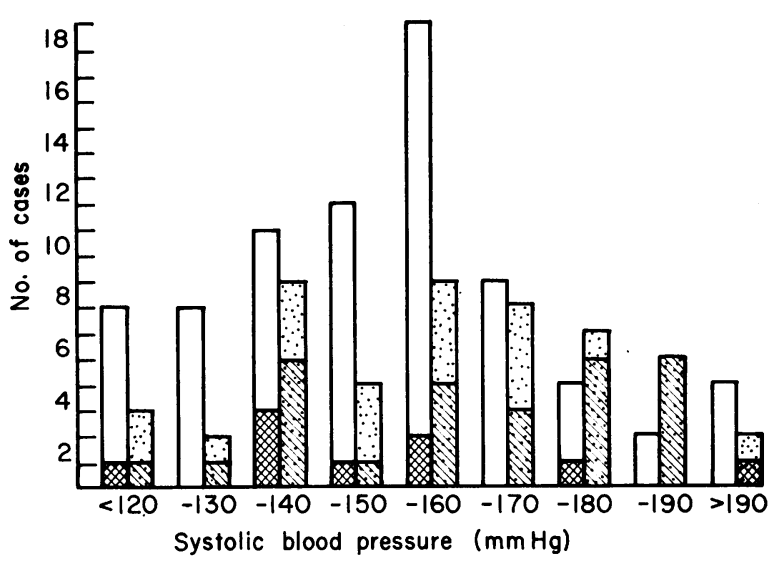

FIG. 2. Systolic blood pressures of 127 thyrotoxic patients without associated heart disease according to heart size and presence of atrial fibrillation. Open columns, Normal heart; stippled columns, enlarged heart; cross-hatched columns, atrial fibrillation.

patients with AF seems to bear no relation to the level of blood pressure. The blood pressure has been measured again in most of the subjects with cardiac enlargement when they were euthy- roid and in eight, four of whom had AF, the diastolic blood pressure was $100 \mathrm{mmHg}$ or more, having been less than this when they presented with thyrotoxicosis. In seven of these there had been no significant change in heart size, whilst in one, the heart was moderately larger after an interval of 3 years.

\section{Discussion}

Enlargement of the heart was present in $35 \%$ of this series of thyrotoxic patients in whom there was no clinical evidence of associated heart disease, and the data also show a progressive increase in the incidence of cardiomegaly with advancing age. Although this is a selected group it is evident that enlargement of the heart is common in older subjects with thyrotoxicosis without an arrhythmia, for AF was present in only half with an enlarged heart. This analysis was retrospective with many of the disadvantages that this entails, and the method of determining heart size is also open to criticism. However, with this large number of patients the findings can reasonably be accepted for if a few have been wrongly classified as having an enlargec heart, it is probable that more have been called $\mathbb{D}$

TABLE 3

Change in heart size in patients with severe cardiac failure

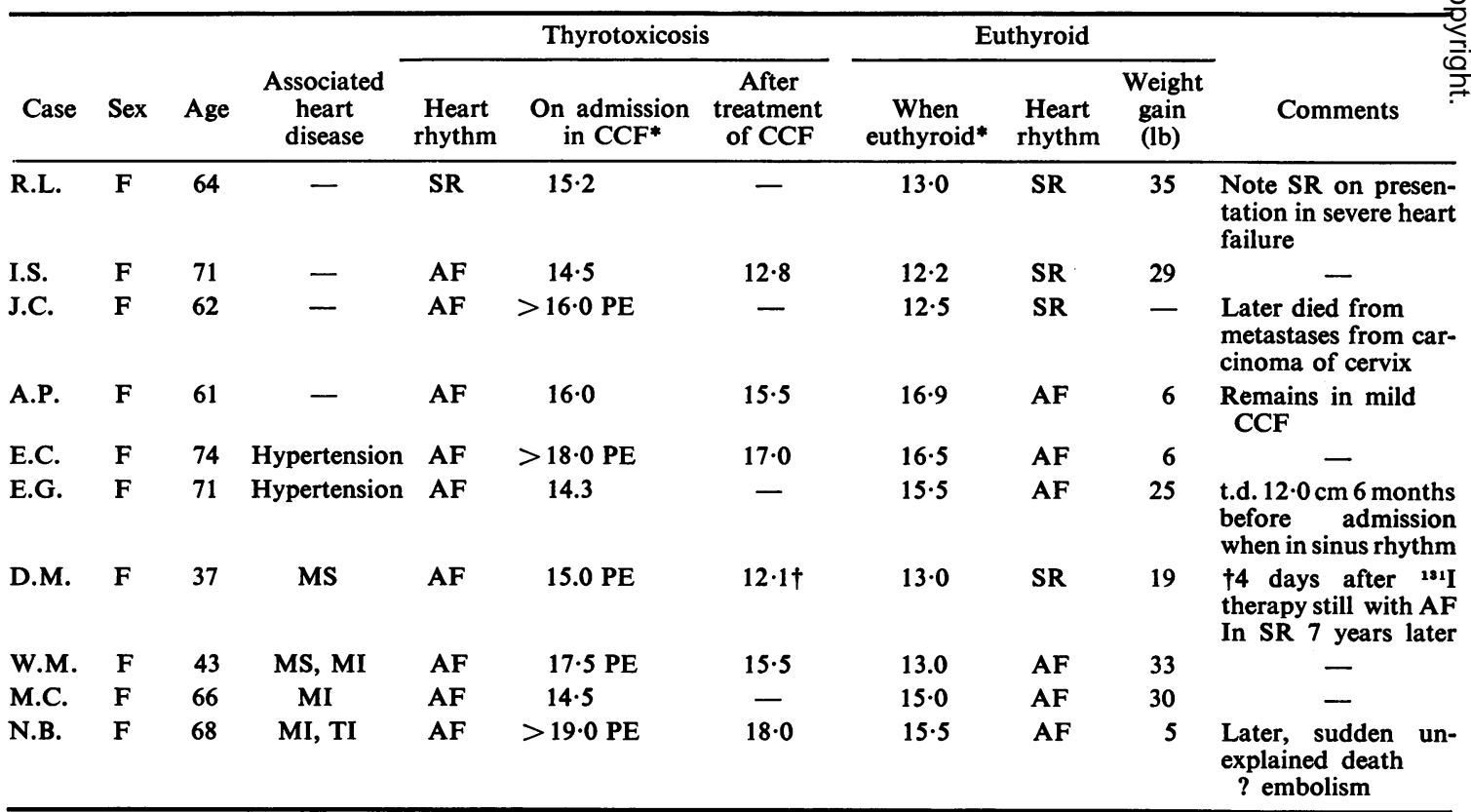

SR = Sinus rhythm; $\mathbf{A F}=$ atrial fibrillation; $\mathbf{C C F}=$ congestive cardiac failure; $\mathbf{P E}=$ pleural effusion; $\mathbf{M S}=$ mitral stenosis; $\mathrm{MI}=$ mitral incompetence; $\mathrm{TI}=$ tricuspid incompetence.

* Transverse diameter of heart (cm). †See item in 'comments'. 
normal when some enlargement was present. Further, a change in the t.d. is accepted as a valid indication of change in heart size when repeated measurements are made on the same individual (Kerley, 1950 ; Massey, 1953).

The reported incidence of cardiac enlargement in thyrotoxicosis varies greatly due to different populations studied and different criteria used for the assessment of heart size and definition of associated heart disease, particularly ischaemic heart disease. Thus Friedberg \& Sohval (1937) considering autopsy material, thought that enlargement of the heart was uncommon without associated heart disease, whereas Parkinson \& Cookson (1931) found cardiac hypertrophy, which involved both ventricles, in more than half of forty autopsies without any evidence of associated heart disease. In living subjects, Parkinson \& Cookson (1931) found radiological evidence of enlargement in $45 \%$ of 130 cases, just under half of whom were in sinus rhythm. Summers \& Surtees (1961) found eighteen instances of uncomplicated enlargement in 200 unselected cases, whereas Sandler \& Wilson (1959) reported only thirteen instances of cardiomegaly without associated heart disease or AF in 462 cases treated with ${ }^{131} I$.

In this series a significant reduction in size of an enlarged heart after treatment of the thyrotoxicosis occurred in eight of forty-four cases without associated heart disease. In four cases there was neither AF nor cardiac failure which shows that effective treatment of thyrotoxicosis alone may result in reduction of size of an enlarged heart. One of our patients developed cardiac enlargement over a period of 6 months with the onset of thyrotoxicosis, her heart reverting to its previous size after treatment. Summers \& Surtees (1961) also reported a patient with a similar observed increase in heart size with the development of thyrotoxicosis though in their case the enlargement persisted after treatment. Parkinson \& Cookson (1931) reported a reduction in heart size in four of eleven subjects after thyroidectomy, two of these had normal rhythm throughout, whilst in the other two AF reverted to sinus rhythm. None of the cases with uncomplicated cardiomegaly reported by Summers \& Surtees (1961) and by Sandler \& Wilson (1959) had a diminution in heart size after treatment.

Atrial fibrillation is usually considered to be the most important cardiac complication of thyrotoxicosis, and the necessity for its prompt and energetic treatment in relation to ${ }^{131} I$ therapy has been stressed elsewhere (Staffurth et al., 1965). It has been found in this series that
AF is uncommon under 55 years in thyrotoxicosis without associated heart disease though enlargement of the heart with sinus rhythm was frequently present between 45 and 55 years. In fact AF was observed in only one of thirteen patients with an enlarged heart who were under 55 years, in comparison with the overall incidence of $48 \%$. It was interesting to find that a reduction in heart size did not occur more often in patients with AF who reverted to sinus rhythm after treatment, and that a reduction in heart size occurred just as often in patients with sinus rhythm throughout. Neither Sandler \& Wilson (1959) nor Summers \& Surtees (1961) make much comment about any change in heart size in their patients with AF after treatment, but it is tacitly assumed that AF itself is the cause of any enlargement of the heart in thyrotoxicosis when there is no associated heart disease. This is certainly so in some cases, particularly when cardiac failure is present, but from our data it would seem probable that in other instances $\mathbf{A F}$ occurs in patients who already have an enlarged heart due to thyrotoxicosis. There was no relationship between $A F$ and the level of the systolic blood pressure in the patients without associated heart disease, though it was relatively more common in our patients with hypertension; however, these were on average older, and it does not appear that hypertension is an important factor in the genesis of AF in thyrotoxicosis, a conclusion also reached by Sandler \& Wilson (1959).

It seems probable that there are several factors operating individually or together in various combinations and with varying importance, that may lead to enlargement of the heart in thyrotoxicosis. These include the severity and length of time the condition has been present, advancing age, AF, masked hypertension and other undiagnosed forms of heart disease. In animals it has been shown that fresh thyroid extract may cause reversible cardiac enlargement (Hewitt, 1920) and we conclude that this may occur in man, though diminution in heart size after treatment is less common than persistent enlargement. Sandler \& Wilson (1959) and Summers \& Surtees (1961) were unable to find any evidence that severity and length of history were important factors in the genesis of thyrotoxic heart disease. Staffurth et al. (1965) looked at the importance of these factors and they found them very difficult to assess. In many instances patients have no idea when the condition started and this even applied to such a definite complication as AF. In general more severe cases will be seen earlier and milder cases after a longer time, so the two 
factors would in any event tend to operate in different directions. In individual cases it certainly seems that one or other of these factors is important.

Age is certainly an important and the most readily recognizable factor in the aetiology of cardiomegaly in thyrotoxicosis for the ageing heart is presumably less able to stand the extra work load even with a normal blood supply. There is no doubt that $\mathbf{A F}$ is an important cause of cardiac enlargement in a proportion of patients but as has been argued above, it is probable that in other instances AF has developed in patients who already have an enlarged heart. Because of the increasing incidence of cardiac complications in older patients it is sometimes considered that other forms of heart disease, particularly coronary artery atherosclerosis, are important factors, even if these cannot be diagnosed. This aspect is well reviewed by Sandler \& Wilson (1959) and we agree with them that thyrotoxicosis alone can certainly cause severe heart disease and we think that unrecognized coronary artery disease is unimportant in the genesis of both cardiomegaly and AF in this condition, partly because neither of these complications was relatively much more common in patients with undoubted ischaemic heart disease (from data included in Fig. 2) and also because we have been struck by the absence of subsequent incidents normally associated with coronary artery disease in the follow-up of our treated patients which in many has now lasted for several years.

The position as regards masked hypertension is more difficult to assess. We have excluded patients with frank hypertension, defined in the same manner as both Sandler \& Wilson (1959) and Summers \& Surtees (1961), from the main series. Fig. 2 shows that there is a tendency for an enlarged heart to be more frequent when the systolic B.P. was more than $160 \mathrm{mmHg}$ but the data are not statistically significant. The systolic B.P. was charted in the histogram because the level of the diastolic B.P. is difficult to assess accurately in the presence of AF and it was desirable to include these patients. The diastolic blood pressure in eight patients with an enlarged heart, but classified as having no associated heart disease, was found to be $100 \mathrm{mmHg}$ or more after treatment. It would appear that in these particular subjects hypertension may well have been present before the onset of thyrotoxicosis, but that the blood pressure was lower on presentation because of the decreased peri- pheral resistance which is part of the condition. In this respect our findings are different from those of Sandler \& Wilson (1959) who concluded that only small and insignificant changes in blood pressure were observed after treatment and that there was no evidence that thyrotoxicosis masked the presence of hypertension. From our material we feel that masked hypertension may be a factor in the causation of cardiac enlargement in a few of our patients, but not in the majority.

All the patients with uncomplicated cardiomegaly reported by Sandler \& Wilson (1959) had left ventricular hypertrophy and this was found in half of the cases of Summers \& Surtees (1961). On the other hand in autopsy material Parkinson \& Cookson (1931) found hypertrophy of both ventricles. We have not found that left ventricular hypertrophy, as judged by ECG, is invariable, though we agree it may be present without hypertension. It would seem that cardiac enlargement, not due to dilatation, is the result of the increased work load in thyrotoxicosis and that in some patients a moderate elevation of blood pressure may be an aggravating g factor. Whatever the full explanation, there is no doubt that enlargement of the heart may be the sole objective sign of thyrotoxic heart disease and when present the thyrotoxicosis should be promptly and energetically treated before furthen complications, particularly AF, occur.

\section{References}

FriedberG, C.K. \& Sohval, A.R. (1937) The occurrence and the pathogenesis of cardiac hypertrophy in Graves' disease. Amer. Heart J. 13, 599.

HewITT, J.A. (1920) The effect of administration of small amounts of thyroid gland on the size and weight of certain organs in the male rat. Quart. J. exp. Physiol. 12, 347.

KERLEY, P. (1950) Text-book of X-Ray Diagnosis (Ed. by C. Shanks and P. Kerley). Lewis, London.

LUSTED, L.B. \& KEATS, T.E. (1967) Atlas of Rontgenographic Measurement. Year Book Medical Publishers, Chicago.

MASSEY, F.C. (1953) Clinical Cardiology. Williams \& Wilkins, Baltimore.

Parkinson, J. \& Cookson, H. (1931) The size and shape of the heart in goitre. Quart. J. Med. 24, 499.

SANDleR, G. \& Wilson, G.M. (1959) The nature and prognosis of heart disease in thyrotoxicosis. Quart.J. Med. 28, 347.

Staffurth, J.S., Gibberd, M.C. \& Hilton, P.J. (1965) Atrial fibrillation in thyrotoxicosis treated with radioiodine. Postgrad. med. J. 41, 663.

STAFFURTH, J.S. \& Young, J. (1967) Delay in control of thyrotoxicosis after treatment with radioactive iodine. J. clin. Endocr. 27, 1062.

Summers, V.K. \& SurTeES, S.J. (1961) Thyrotoxicosis and heart disease. Acta med. scand. 169, 661 .

WoOD, P. (1961) Diseases of the Heart and Circulation. Eyre \& Spottiswoode, London. 